\title{
The Warburg Effect as a Type B Lactic Acidosis in a Patient With Acute Myeloid Leukemia: A Diagnostic Challenge for Clinicians
}

\author{
Clément Brault ${ }^{1 * t}$, Yoann Zerbib ${ }^{1 \dagger}$, Caroline Delette ${ }^{2}$, Julien Marc ${ }^{1}$, Bérengère Gruson ${ }^{2}$, \\ Jean P. Marolleau ${ }^{2}$ and Julien Maizel ${ }^{1}$ \\ ${ }^{1}$ Réanimation Médicale, CHU Amiens-Picardie, Amiens, France, ${ }^{2}$ Hématologie Clinique, CHU Amiens-Picardie, Amiens, \\ France
}

OPEN ACCESS

Edited by:

Sara Galimberti,

Università degli Studi di

Pisa, Italy

Reviewed by:

Michael Diamantidis,

University Hospital of

Larissa, Greece

Basem M. William,

The Ohio State University,

United States

Giuseppe Alberto Palumbo,

Università degli Studi di Catania, Italy

*Correspondence:

Clément Brault

brault.clement@chu-amiens.fr

tThese authors have contributed equally to this work.

Specialty section:

This article was submitted to Hematology Oncology, a section of the journal

Frontiers in Oncology

Received: 11 March 2018

Accepted: 06 June 2018

Published: 20 June 2018

Citation:

Brault C, Zerbib Y, Delette C, Marc J,

Gruson B, Marolleau JP and Maizel J

(2018) The Warburg Effect as a

Type B Lactic Acidosis in a Patient

With Acute Myeloid Leukemia:

A Diagnostic Challenge

for Clinicians.

Front. Oncol. 8:232.

doi: $10.3389 /$ fonc. 2018.00232
Introduction: The Warburg effect (WE) is an uncommon cause of type B lactic acidosis $(\llcorner A)$ due to a deregulation of carbohydrate metabolism in neoplastic cells where lactic fermentation predominates over oxidative phosphorylation regardless of the oxygen level.

Case presentation: We report the case of a 57-year-old man presenting with concomitant acute myeloid leukemia and type B LA with asymptomatic hypoglycemia. We did not find arguments for a septic state, liver dysfunction, or acute mesenteric ischemia. The WE was suspected, and chemotherapy was immediately undertaken. We observed a rapid and sustained decrease in lactate level and normalization of blood glucose. Unfortunately, we noted a relapse of acute leukemia associated with WE soon after treatment initiation and the patient died in the Intensive Care unit.

Discussion: Some patients may present complications directly related to an underlying hematological malignancy. The WE is one of these complications and should be suspected in patients with both hypoglycemia and LA. We propose a checklist in order to help clinicians manage this life-threatening complication. Before considering WE, clinicians should eliminate diagnoses such as septic shock or mesenteric ischemia, which require urgent and specific management.

Conclusion: The diagnosis of WE can be challenging for clinicians in the Hematology department and the Intensive Care unit. Prompt diagnosis and rapid, adapted chemotherapy initiation may benefit patient survival.

Keywords: lactic acidosis, hypoglycemia, the Warburg effect, acute leukemia, intensive care unit, chemotherapy

\section{INTRODUCTION}

Lactic acidosis (LA) is defined as a pH of less than 7.35 and a blood lactate level greater than $5 \mathrm{mmol} / \mathrm{L}$ (1). Type A LA is related to inadequate tissue perfusion in a context of sepsis, circulatory failure, hypovolemia, or severe hypoxemia. Conversely, type B LA occurs without any sign of organ dysfunction and is related to a deregulation of cellular metabolism (1-5).

Lactic acidosis is a common and serious complication at the onset or during the management of hematological malignancies, most often related to type A LA. A recent study including 372 patients 
with hematological malignancies admitted to the Intensive Care unit reported 58 cases of LA, among which only six cases were type B LA (6).

The Warburg effect (WE), or hyper-Warburgism, is a classic but challenging cause of type B LA in hematological malignancies. The WE is due to a specific malignant-cell metabolism, where lactic fermentation predominates over oxidative phosphorylation regardless of the oxygen level (7). It has mostly been associated with patients with lymphoma, but also with those with acute leukemia, chronic lymphocytic leukemia, chronic myelomonocytic leukemia, or multiple myeloma $(1,5,8)$. We report the case of a 57-year-old man who developed WE as a consequence of acute myeloid leukemia (AML). We propose a checklist to assist clinicians in the management of this lifethreatening complication.

\section{CASE PRESENTATION}

A 57-year-old man presented with neutropenia, since May 2016 due to a myelodysplastic syndrome. The revised international prognostic scoring system was 0 , and no specific treatment was undertaken. Other significant past medical history included well-controlled hypertension treated with quinapril and type 2 diabetes mellitus without medication.

In November 2016, he experienced severe asthenia and excessive sweating. Laboratory tests revealed leukocyte count of 8,000 per cubic millimeter with hyperblastosis (23\%), anemia, and thrombocytopenia. The results of the bone marrow aspiration confirmed AML (M4 type) according to the French-American-British classification, without extramedullary manifestations. FLT3, CEBP $\alpha$, and NPM1 were not mutated and no cytogenetic abnormalities were found. This AML was secondary to a myelodysplastic syndrome with single lineage dysplasia. For these reasons, the patient was eligible for a hematopoietic stem cell allograft.

In the Hematology department, an asymptomatic hypoglycemia that persists despite glucose infusion was found. Laboratory tests showed type B LA with an elevated blood lactate of $14 \mathrm{mmol} / \mathrm{L}$ (normal range, $0.5-2 \mathrm{mmol} / \mathrm{L}$ ) associated with a slightly decreased $\mathrm{pH}$ of 7.35 (normal range, 7.38-7.42). Serum bicarbonate was low at $13 \mathrm{mmol} / \mathrm{L}$ (normal range, $24-32 \mathrm{mmol} / \mathrm{L}$ ) with normal renal function tests and an elevated anion gap of $28 \mathrm{mmol} / \mathrm{L}$. Liver function tests were normal.

The patient was transferred to the Intensive Care unit. His temperature was $37.7^{\circ} \mathrm{C}$, his blood pressure was normal at $149 / 82 \mathrm{mmHg}$, his pulse was $119 \mathrm{bpm}$, and the respiratory rate was 28 per minute without respiratory distress which indicated Kussmaul breathing. The patient did not present any signs of hypoperfusion (he had normal blood pressure, absence of mottling, normal capillary refilling test). The palpation of the abdomen was normal without diarrhea. In the absence of a type A LA etiology, and in the context of AML, we suspected WE. Chemotherapy by doxorubicin $60 \mathrm{mg} / \mathrm{m}^{2}$ and cytarabine $100 \mathrm{mg} / \mathrm{m}^{2}$ was initiated at day 1 . A slight tumor lysis syndrome was observed without kidney injury. We noted no recurrence of hypoglycemia, a rapid normalization of $\mathrm{pH}$, and a decrease in the blood lactate level: $12.1 \mathrm{mmol} / \mathrm{L}$ at day $2,9.2 \mathrm{mmol} / \mathrm{L}$ at day 3, $2.6 \mathrm{mmol} / \mathrm{L}$ at day 5 , and $1.6 \mathrm{mmol} / \mathrm{L}$ at day 7 (Figure 1). We observed no further complications, allowing ICU discharge at day 8 .

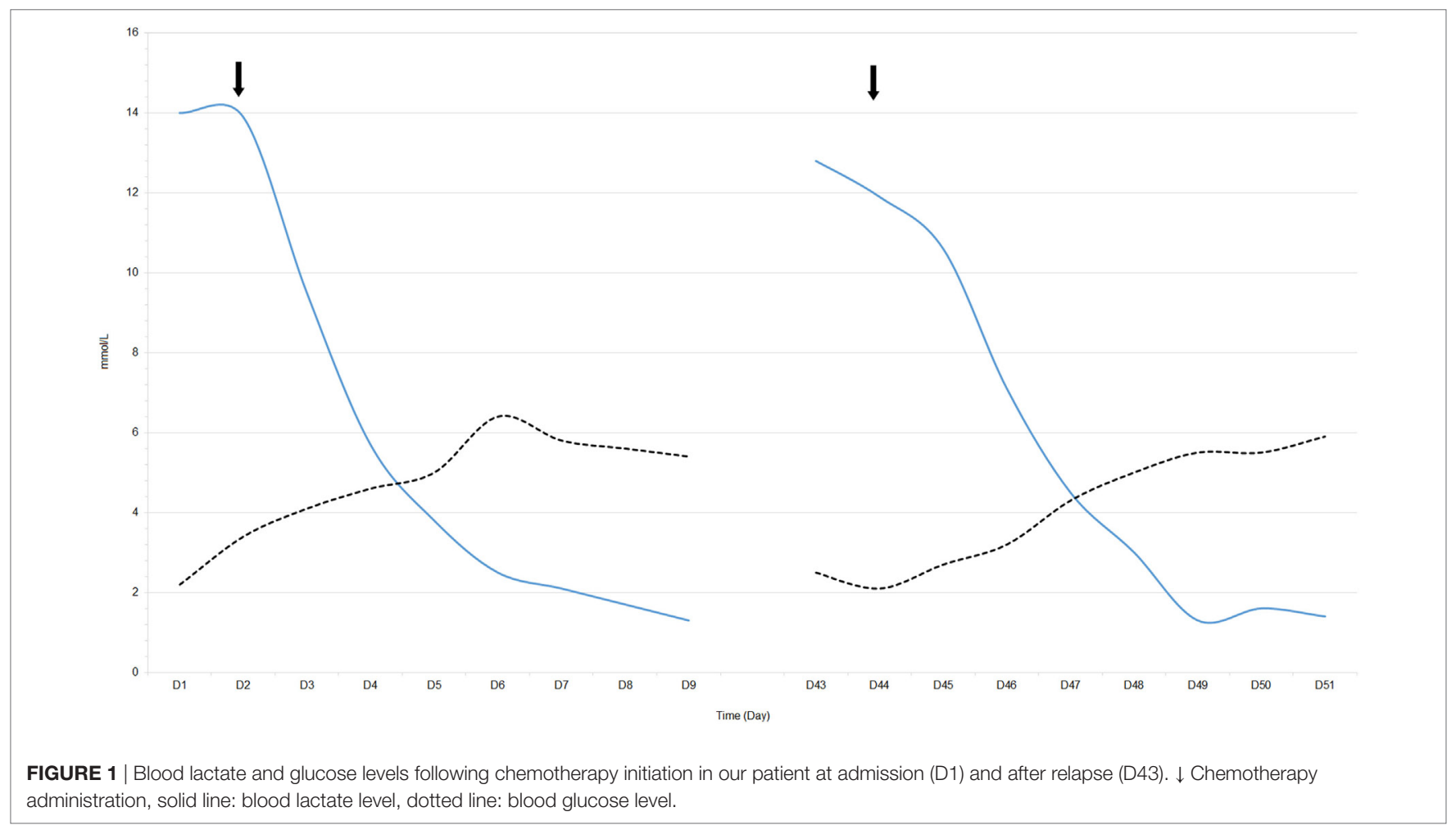


Unfortunately, a relapse of AL occurred at day 43. This relapse was associated with a new hyperlactatemia $(\mathrm{pH}: 7.37$ and blood lactate level: $12.8 \mathrm{mmol} / \mathrm{L}$ ) and asymptomatic hypoglycemia related to a recurrence of WE (Figure 1). Salvage chemotherapy with cytarabine $1,500 \mathrm{mg} / \mathrm{m}^{2}$ and gemtuzumab $3 \mathrm{mg} / \mathrm{m}^{2}$ was initiated, resulting in the disappearance of WE. However, the patient later developed septic shock as a complication of chemotherapy-induced aplasia and digestive infection. We suspected infectious colitis because of diarrhea and abdominal guarding. Unfortunately, the CT scan was not contributory and we could not perform a colonoscopy to confirm the diagnosis. Empirical broad-spectrum antibiotic therapy with imipenem, vancomycin, and gentamycin was administered. He eventually died in the Intensive Care unit in a state of multiple organ dysfunction due to the septic shock.

\section{DISCUSSION}

Here, we reported the case of an AML complicated by a WE, an uncommon cause of type-B LA. In aerobic conditions, oxidative phosphorylation by the mitochondria is the main source of cellular energy, with the delivery of 38 units of adenosine triphosphate (ATP) per molecule of glucose. In anaerobic conditions, the mitochondrial respiratory chain complex is non-functional, so the energy supply is provided by lactic fermentation, with the delivery of only two units of ATP per molecule of glucose and some degree of lactate accumulation. The WE is a specific malignant-cell metabolism (in hematological malignancies and solid neoplasms) where lactic fermentation predominates over oxidative phosphorylation, regardless of the oxygen level $(9,10)$. Mechanisms explaining WE in neoplastic cells are misunderstood, but depend on the microenvironmental supply of oxygen, overexpression of factors promoting the lactic fermentation pathway (e.g., hexokinase, insulin-like growth factors, or hypoxiainducible factor $1 \alpha$ ), or inflammatory cytokines (e.g., tumor necrosis factor alpha) $(10,11)$. Recently, oncogenes like FLT3 tyrosine kinase, K-ras, c-Myc, and Bcr-Abl have been shown to induce WE promoting glycolysis or mitochondrial dysfunction (12). However, the search for the mutation in FLT3 tyrosine kinase, usually detected in $30 \%$ of AML, was negative in our patient. The WE, combined with the rapid growth and replication of tumor cells and impairment of lactate clearance by the liver or kidneys, can explain blood lactate accumulation and occurrence of metabolic acidosis (6).

In the presence of LA in patients with hematological malignancies, clinicians should evoke different etiologies. It should be borne in mind that mechanisms responsible for LA in patients with malignancies are not mutually exclusive and can co-exist (Figure 2).

Classic causes are hemodynamic instability associated with hypoperfusion (mean arterial blood pressure lower than $65 \mathrm{mmHg}$ or use of catecholamine, mottling, prolonged capillary refilling time) or severe hypoxemia. Many etiologists of hemodynamic instability exist, but particular attention should be paid to infections (especially pneumonia or gastro-intestinal infections) in these immunocompromised patients (13).
Regional tissue ischemia can also be responsible for LA. The diagnosis of mesenteric ischemia should be considered, keeping in mind that performance of clinical and radiological signs are limited. Endoscopic explorations of the digestive tract, which can help us to confirm or exclude this diagnosis, are often difficult to carry out because of the high frequency of thrombocytopenia or coagulopathy associated with malignancies. Coral or tumor thrombi, vascular mesenteric infiltration, and extrinsic vascular compression are the main mechanisms leading to mesenteric ischemia (14).

Hepatic infiltration by lymphoma or leukemia can lead to lactate clearance impairment. This involvement is frequent, but rarely causes acute and severe hepatic failure with LA. Liver biopsy is often necessary for diagnosis because of the lack of specificity of radiologic signs. The progression of the disease is often fatal despite chemotherapy $(2,15)$. Similarly, renal failure due to tumor lysis syndrome is common in hematological malignancies and may contribute, to a lesser extent, to lactate accumulation (16).

Finally, less frequent LA causes can be considered: hyperosmolar hyperglycemic states or other complications of diabetes mellitus, nutritional deficiency (e.g., thiamine deficiency), anaerobic muscle activity, and drugs or toxins. Among drugs implicated, metformin or nucleoside reverse transcriptase inhibitors are the most common (Table 1). More rarely in adult patients, some hereditary enzymatic defects (e.g., glucose6-phosphatase deficiency) resulting in type B LA have been described $(2-5,17)$.

A literature review found only five patients with AML associated with type B LA $(5,18-21)$. Among them, lactate clearance impairment was suspected in three patients because of a history of liver cirrhosis (5), and a possible liver infiltration by leukemia cells as well as severe renal failure were also found $(18,19)$. Thiamine deficiency was proved in one patient (20). This illustrates that several mechanisms leading to hyperlactatemia can co-exist in the same patient.

Clinical examination is nonspecific in WE. Tachypnea without hypoxemia relevant to LA-Kussmaul breathing-is often on the forefront of clinical attention. Severe hypoglycemia is classic and evocative, but not always present. It is completely asymptomatic because lactate is used by the brain as a source of energy by a misunderstood mechanism involving astrocytic and neuronal cells. This specificity explains the absence of fluorodeoxyglucose uptake in the brain in positron emission tomography/computed tomography studies of patients with WE. Hypoglycemia is often not correctable by dextrose infusion (22). Abnormal laboratory findings frequently include a very high blood lactate level $(>10 \mathrm{mmol} / \mathrm{L})$, contrasting with the absence of signs of tissue hypoperfusion (6).

Since there is no randomized controlled trial, we can hardly provide any specific and definite recommendations for WE management. Nevertheless, a fast diagnostic workup of WE and the understanding of the implication of the underlying malignancy in the metabolic disorder seems to be essential. This implies that chemotherapy administration is the main therapeutic action for fast recovery, with the aim of disrupting lactate production by tumor cells (1). Regression of hyperlactatemia is 


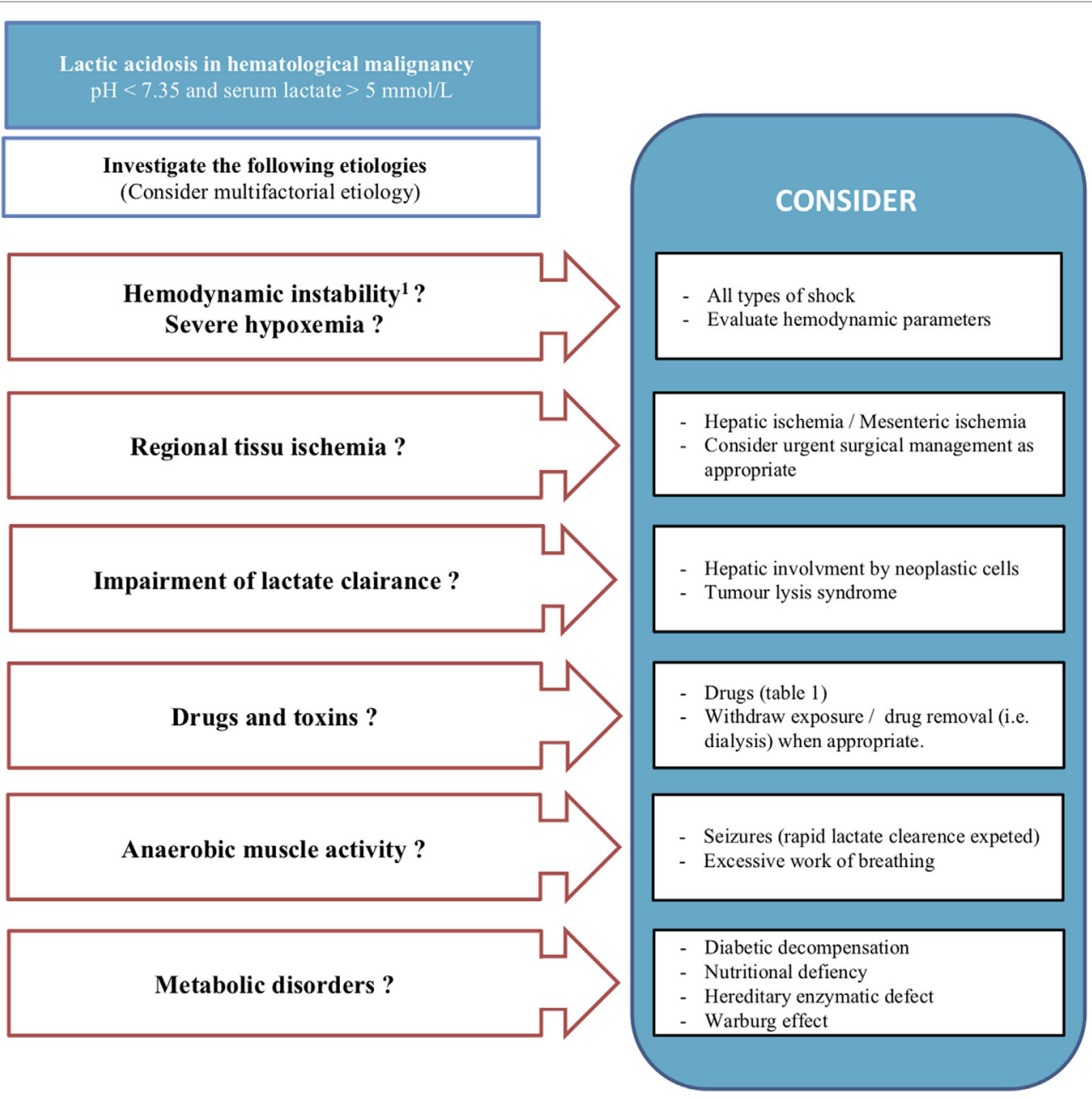

FIGURE 2 | LA in hematological malignancy: a checklist for clinicians. Mechanisms responsible for LA are not mutually exclusive and can co-exist in the same patient. ${ }^{1}$ Mean arterial blood pressure $<65 \mathrm{mmHg}$ or use of vasoactive agent, mottling, or prolonged capillary refilling test. LA, lactic acidosis.

TABLE 1 | Drugs associated with type B lactic acidosis.

\section{Drugs}

\section{Acetaminophen}

Antiretroviral therapy (e.g., stavudine, zidovudine, and lamivudine)

$\beta$-2 agonists (e.g., salbutamol, terbutaline)

Biguanides (e.g., metformin, phenformin)

Isoniazid

Lactulose

Linezolid

Nalidixic acid

Niacin

Nitroprusside sodium

Propofol

Simvastatin

Theophylline

Vasoactive drugs (e.g., epinephrine, norepinephrine)

the general rule and is a good retrospective argument for WE diagnosis (21).

Other therapeutic options can be discussed, but lack solid and relevant arguments. Renal replacement therapy has been initiated in several cases with no effect on the lactate level, probably because of inadequate lactate clearance compared to lactate production $(1,23)$. Thus, the impact of renal replacement therapy on WE remains questionable. Sodium bicarbonate infusion has also been used for its proton binding property, but we can hardly support this strategy because of potential complications (e.g., intracellular acidification, volume overload) (23). In addition, some authors have tested empirical thiamine infusion for the potential benefit for mitochondrial function without severe risks (1). Thiamine deficiency could occur in patients with malignancies, explained by exclusive parenteral nutrition without thiamine supplementation (5). Moreover, some chemotherapy (e.g., methotrexate) may limit the cellular bioavailability of thiamine (24). Thiamine plays a key role as a pyruvate dehydrogenase enzyme cofactor, promoting the aerobic glycosylation pathway (25). Therefore, we recommend routine thiamine supplementation.

In our experience, WE occurs when there is a high tumor burden with a high proliferation rate, often complicated by an induced tumor lysis syndrome $(26,27)$. Therefore, the presence of WE in a patient receiving hematological care should be considered as having a major risk factor for tumor lysis syndrome and 
should lead clinicians to initiate prophylactic treatment. Patient survival is determined by the body's ability to correct the metabolic acidosis induced by hyperlactatemia. This compensation is mainly related to alveolar hyperventilation. The excessive effort relative to the strength and endurance of the respiratory muscles can induce respiratory failure resulting in a sudden loss of compensation for acidosis. Data on the mortality of WE patients are limited, but type B LA seems to be a poor prognostic factor of survival in case series (6-8).

\section{CONCLUSION}

Type B LA is a challenging situation for clinicians and can indicate complications of malignancies. The WE is a rare but severe condition that needs to be recognized. Clinicians should suspect WE in patients with hematological malignancies who develop both type B LA and acute asymptomatic hypoglycemia. The management of such specific complications relies on urgent initiation of chemotherapy along with life support management.

\section{REFERENCES}

1. Gardner AJ, Griffiths J. A case of type B lactic acidosis as a complication of chronic myelomonocytic leukaemia: a case report and review of the literature. J Med Case Rep (2015) 9(1):16-20. doi:10.1186/1752-1947-9-16

2. Sillos EM, Shenep JL, Burghen GA, Pui CH, Behm FG, Sandlund JT. Lactic acidosis: a metabolic complication of hematological malignancies: case report and review of the literature. Cancer (2001) 92(9):2237-46. doi:10.1002/ 1097-0142(20011101)92:9<2237::AID-CNCR1569>3.0.CO;2-9

3. Kraut JA, Madias NE. Lactic acidosis. N Engl J Med (2014) 371(12):2309-19. doi:10.1056/NEJMra1309483

4. Andersen LW, Mackenhauer J, Roberts JC, Berg KM, Cocchi MN, Donnino MW. Etiology and therapeutic approach to elevated lactate levels. Mayo Clin Proc (2013) 88(10):1127-40. doi:10.1016/j.mayocp.2013.06.012

5. Friedenberg AS, Brandoff DE, Schiffman FJ. Type B lactic acidosis as a severe metabolic complication in lymphoma and leukemia: a case series from a single institution and literature review. Medicine (2007) 86(4):225-32. doi:10.1097/ MD.0b013e318125759a

6. De Raes EA, Benoit DD, Depuydt PO, Offner F, Nollet J, Vantilborgh AK, et al. Early recognition of malignant lactic acidosis in clinical practice: report on 6 patients with haematological malignancies. Acta Clin Belg (2012) 67(5):347-51. doi:10.2143/ACB.67.5.2062688

7. Claudino WM, Dias A, Tse W, Sharma VR. Type B lactic acidosis: a rare but life threatening hematological emergency. A case illustration and brief review. Am J Blood Res (2015) 5(1):25-9.

8. Ruiz JP, Singh AK, Hart P. Type B lactic acidosis secondary to malignancy: case report, review of published cases, insights into pathogenesis, and prospects for therapy. ScientificWorldJournal (2011) 11:1316-24. doi:10.1100/ tsw.2011.125

9. Devic S. Warburg effect - a consequence or the cause of carcinogenesis? I Cancer (2016) 7(7):817-22. doi:10.7150/jca.14274

10. Vander Heiden MG, Cantley LC, Thompson CB. Understanding the Warburg effect: the metabolic requirements of cell proliferation. Science (2009) 324(5930):1029-33. doi:10.1126/science.1160809

11. Razungles J, Cavaillès V, Jalaguier S, Teyssier C. [The Warburg effect: from theory to therapeutic applications in cancer]. Med Sci (2013) 29(11):1026-33. doi:10.1051/medsci/20132911020

12. Ju HQ, Zhan G, Huang A, Sun Y, Wen S, Yang J. ITD mutation in FLT3 tyrosine kinase promotes Warburg effect and renders therapeutic sensitivity to glycolytic inhibition. Leukemia (2017) 31(10):2143-50. doi:10.1038/leu. 2017.45

13. Cannas G, Pautas C, Raffoux E, Quesnel B, de Botton S, de Revel T, et al. Infectious complications in adult acute myeloid leukemia: analysis of the Acute Leukemia French Association-9802 prospective multicenter clinical

\section{CONSENT FOR PUBLICATION}

Written informed consent was obtained from the next of kin for the publication of this case report.

\section{ETHICS STATEMENT}

Ethic approval and consent to participate: according to the French legislation, no ethics approval was necessary. Consent for publication: because of the death of the patient, only the consent of his wife could be obtained.

\section{AUTHOR CONTRIBUTIONS}

$\mathrm{CB}$ and $\mathrm{YZ}$ have an equal contribution. $\mathrm{CB}, \mathrm{YZ}$ and JMaizel wrote the manuscript. CD, BG, JMarc and JPM had a critical review of the manuscript. All authors read and approved the final manuscript.

trial. Leuk Lymphoma (2012) 53(6):1068-76. doi:10.3109/10428194.2011. 636812

14. Cudnik MT, Darbha S, Jones J, Macedo J, Stockton SW, Hiestand BC. The diagnosis of acute mesenteric ischemia: a systematic review and metaanalysis. Acad Emerg Med (2013) 20(11):1087-100. doi:10.1111/acem. 12254

15. Tomasian A, Sandrasegaran K, Elsayes KM, Shanbhogue A, Shaaban A, Menias CO. Hematological malignancies of the liver: spectrum of disease. Radiographics (2015) 35(1):71-86. doi:10.1148/rg.351130008

16. Tiefenthaler M, Amberger A, Bacher N, Hartmann BL, Margreiter R, Kofler R, et al. Increased lactate production follows loss of mitochondrial membrane potential during apoptosis of human leukaemia cells. Br J Haematol (2001) 114(3):574-80. doi:10.1046/j.1365-2141.2001.02988.x

17. Fall JP, Szerlip HM. Lactic acidosis: from sour milk to septic shock. J Intensive Care Med (2005) 20(5):255-71. doi:10.1177/0885066605278644

18. Dogan E, Erkoc R, Sayarlioglu H, Alici S, Dilek I, Alici O. Fatal lactic acidosis due to leukemic transformation in a patient with non-Hodgkin's lymphoma: case report. Adv Ther (2005) 22(5):443-6. doi:10.1007/BF02849863

19. Ouchikhe A, Le Bivic JL, Longuet O, Maindivide J, Vincent JF. [Severe lactic acidosis revealing hematologic malignancy]. Ann Fr Anesth Reanim (2014) 33(6):430-2. doi:10.1016/j.annfar.2014.05.005

20. Rovelli A, Bonomi M, Murano A, Locasciulli A, Uderzo C. Severe lactic acidosis due to thiamine deficiency after bone marrow transplantation in a child with acute monocytic leukemia. Haematologica (1990) 75(6):579-81.

21. Udayakumar N, Rajendiran C, Muthuselvan R. A typical presentation of acute myeloid leukemia. JCancer Res Ther (2006) 2(2):82-4. doi:10.4103/ 0973-1482.25858

22. Elhomsy GC, Eranki V, Albert SG, Fesler MJ, Parker SM, Michael AG, et al. "Hyper-warburgism," a cause of asymptomatic hypoglycemia with lactic acidosis in a patient with non-Hodgkin's lymphoma. J Clin Endocrinol Metab (2012) 97 (12):4311-6. doi:10.1210/jc.2012-2327

23. Kraut JA, Madias NE. Lactic acidosis: current treatments and future directions. Am J Kidney Dis (2016) 68(3):473-82. doi:10.1053/j.ajkd.2016. 04.020

24. Zhao R, Gao F, Wang Y, Diaz GA, Gelb BD, Goldman ID. Impact of the reduced folate carrier on the accumulation of active thiamine metabolites in murine leukemia cells. J Biol Chem (2001) 276(2):1114-8. doi:10.1074/jbc. M007919200

25. Masood U, Sharma A, Nijjar S, Sitaraman K. B-cell lymphoma, thiamine deficiency, and lactic acidosis. Proc (Bayl Univ Med Cent) (2017) 30(1):69-70. doi:10.1080/08998280.2017.11929534

26. Cairo MS, Bishop M. Tumour lysis syndrome: new therapeutic strategies and classification. Br J Haematol (2004) 127(1):3-11. doi:10.1111/j.1365-2141. 2004.05094.x 
27. Chan FH, Carl D, Lyckholm LJ. Severe lactic acidosis in a patient with B-cell lymphoma: a case report and review of the literature. Case Rep Med (2009) 2009:534-61. doi:10.1155/2009/534561

Conflict of Interest Statement: The authors declare that the research was conducted in the absence of any commercial or financial relationships that could be construed as a potential conflict of interest.
Copyright (® 2018 Brault, Zerbib, Delette, Marc, Gruson, Marolleau and Maizel. This is an open-access article distributed under the terms of the Creative Commons Attribution License (CC BY). The use, distribution or reproduction in other forums is permitted, provided the original author(s) and the copyright owner are credited and that the original publication in this journal is cited, in accordance with accepted academic practice. No use, distribution or reproduction is permitted which does not comply with these terms. 